\title{
Validation of first-order diffraction theory for the traveltimes and amplitudes of propagating waves
}

\author{
Jeroen Jocker ${ }^{1}$, Jesper Spetzler ${ }^{2}$, David Smeulders ${ }^{2}$, and Jeannot Trampert ${ }^{3}$
}

\begin{abstract}
Ultrasonic measurements of acoustic wavefields scattered by single spheres placed in a homogenous background medium (water) are presented. The dimensions of the spheres are comparable to the wavelength and the wavelength and represent both positive (rubber) and negative (teflon) velocity anomalies with respect to the background medium. The sensitivity of the recorded wavefield to scattering in terms of traveltime delay and amplitude variation is investigated. The results validate a linear (first-order) diffraction theory for wavefields propagating in heterogeneous media with anomaly contrasts on the order of $\pm 15 \%$. The diffraction theory is compared further with the exact results known from literature for scattering from an elastic sphere, formulated in terms of Legendre polynomials. To investigate the $2 \mathrm{D}$ case, the firstorder scattering theory is tested against 2D elastic finite-difference calculations. As the presented theory involves a volume integral, it is applicable to any geometric shape, and the scattering object does not need to be spherical or any other specific symmetrical shape. Furthermore, it can be implemented easily in seismic data inversion schemes, which is illustrated with examples from seismic crosswell tomography and a reflection experiment.
\end{abstract}

\section{INTRODUCTION}

The characteristics of finite-frequency waves propagating in heterogeneous media are fundamentally different from what is predicted by standard ray theory. Ray theory is a high-frequency solution for the wave equation, which means that energy travels along lines in space joining the source and receiver. It is correct to use ray theory to model high-frequency wave propagation in simple media consisting of large-scale velocity structures. On the other hand, the high-frequency approximation in ray theory breaks down for finite-frequency waves propagating in complex media with velocity structures smaller than the wavelength and the Fresnel volume. The Fresnel volume is the region between the source and receiver in which the scattered wavefield contributes constructively at the receiver position; i.e., arrival times of scattered waves differ by less than half a period from the direct wave. In such heterogeneous media, diffraction effects become important so that the phase and amplitude variation of propagating waves behave so that cannot be explained by the geometric ray approximation.

We present an overview of a first-order finite-frequency wave theory for heterogeneous media for which ray theory is invalid. In brief, a frequency-dependent term for the single-scattering process (i.e., a first-order approach) is added to the standard point-source solution for a transmitted wavefield. New ultrasonic waveform data are recorded in an experimental setting where a sphere of teflon or rubber is located in a reference water medium. The ultrasonic experiment allows us to clearly illustrate the fundamental differences between a finite-frequency wave theory and ray theory. The considered theory is limited to contrasts in the compressional speed of sound only, and no density contrasts are considered. With respect to the ultrasonic measurements, this means that density contrasts as well as shear wave effects are neglected. The influence of these omissions is studied through comparison with the exact solution for scattering of an incident field because of the presence of an elastic sphere (Hickling, 1962).

In the frequency-dependent single-scattering approach, the maximum traveltime sensitivity to velocity anomalies is off the geometric ray, whereas the amplitude has a significant sensitivity along the ray path. In ray theory, these sensitivities appear to be quite different, but in the high-frequency limit, the scattering kernels converge to the ray theoretical ones. Hung et al. (2001) performed a 3D numerical wave experiment with a smooth velocity anomaly. They used a pseudospectral method to solve the $3 \mathrm{D}$ acoustic wave equation, and the

Manuscript received by the Editor June 8, 2005; revised manuscript received April 28, 2006; published online November 3, 2006.

${ }^{1}$ Formerly Delft University of Technology, Department of Geotechnology, The Netherlands; presently Schlumberger-Doll Research, 36 Old Quarry Road, Ridgefield, Connecticut 06877.E-mail: jjocker@ ridgefield.oilfield.slb.com.

${ }^{2}$ Delft University of Technology, Department of Geotechnology, P.O. Box 5028, 2600 GA Delft, The Netherlands. E-mail: j.spetzler@tudelft.nl; d.m.j.smeulders@tudelft.nl.

${ }^{3}$ Utrecht University, Faculty of Geosciences, P.O. Box 80021, 3508 TA Utrecht, The Netherlands. E-mail: jeannot@ geo.uu.nl. (C) 2006 Society of Exploration Geophysicists. All rights reserved. 
smoothness of the velocity perturbation was a numerical requirement. In our experiment, the effect of sharp discontinuities on real data is investigated. As real experiments are inherently $3 \mathrm{D}$, the $2 \mathrm{D}$ case, which has fundamentally different finite-frequency kernels, is investigated numerically.

The applied finite-frequency wave theory is well documented by de Wolf (1967), Aki and Richards (1980), Rytov et al., (1989), Woodward (1992), Snieder and Lomax (1996), and Spetzler and Snieder $(2001 ; 2004)$. The latter used the Rytov approximation to include the effect of heterogeneous small-scale structures on the phase of propagating waves. Born and Wolf (1959), Marquering et al. (1999), Zhao et al. (2000), and Hung et al. (2001) chose to work with the Born approximation to account for single-scattering effects on the traveltime of propagating waves. Aki and Richards (1980), Woodward (1992), and Dahlen and Baig (2002) focused on the finite-frequency wave theory for amplitude variations. De Hoop (1985) showed an elegant derivation of the first Born approximation for plane waves where the geometric shape of the contrast term is explicitly included. Beydoun and Tarantola (1988) tested the validity of the Born and Rytov approximation for a 1D layered medium and found that the Rytov approach is most suitable for transmitted waves, whereas for reflected waves, the Born approximation is recommended.

The finite-frequency wave theory we use for traveltimes and amplitudes takes first-order scattering effects into account, and it is thus linear with respect to velocity perturbations. However, depending on the complexity in terms of shape and strength of velocity anomalies, nonlinear ray-bending effects become increasingly important when triplications occur in the wavefield. We have recorded 3D real ultrasonic waveform data with (teflon) and without (rubber) triplications. We supplement the 3D real ultrasonic data experiment with 2D numerical simulations. The numerical study yields the possibility to estimate the range of validity of the linear finite-frequency wave theory in the regime of weakly developed triplications.

In this paper, we present and validate a simple first-order scattering theory for wave propagation in the presence of objects with dimensions comparable to the wavelength, but smaller in size than the Fresnel zone. Because of the linearity between the traveltimes and amplitude attributes and the velocity perturbation field, it is easy to implement the presented wave theory in seismic imaging experiments. Anomalies do not need to be a specific shape, as the theory involves a general volume integral over the velocity perturbation field. To illustrate this, two applications of the tested wave-scattering theory in exploration seismic experiments are discussed. The first example is crosswell tomography, and the second example is from reflection seismic.

In the following, we give first an overview of the theory for traveltime and amplitude variations of finite-frequency wavefields. Next, the 3D ultrasonic wave experiment and theory validation section are presented, followed by a section on the $2 \mathrm{D}$ numerical simulations. We conclude after discussing the two possible seismic applications for the proposed wave theory.

\section{THEORY}

In this section, the theory is presented for finite-frequency waves propagating in heterogeneous media with anomalies smaller than the Fresnel zone. The wavefield is modeled using the first-order Rytov approximation. The Rytov approximation only describes contrasts in sound speed (not in density) and neglects any shear-wave ef- fects. The importance of these approximations will be investigated by comparing the exact solution for a point-source wavefield incident upon an elastic sphere in a fluid. First, we derive expressions for traveltime shifts and amplitude variations for the 2D case, followed by a generalization to three dimensions.

In equation 1, the Rytov wavefield $P_{R}\left(\mathbf{r}_{r}, \mathbf{r}_{s}, \omega\right)$ at the angular frequency $\omega=2 \pi f$ emitted from the source position $\mathbf{r}_{s}$ and recorded at the receiver position $\mathbf{r}_{r}$ is given by

$$
P_{R}\left(\mathbf{r}_{r}, \mathbf{r}_{s}, \omega\right)=P_{0}\left(\mathbf{r}_{r}, \mathbf{r}_{s}, \omega\right) \exp \left(\frac{P_{B}}{P_{0}}\left(\mathbf{r}_{r}, \mathbf{r}_{s}, \omega\right)\right),
$$

which accounts for the single-scattering process of a propagating wavefield in heterogeneous media (Born and Wolf, 1959; Aki and Richards, 1980; Snieder and Lomax, 1996). In equation 1 and in all subsequent derivations, $P$ is defined as a pressure field. The reference wavefield inherent to the reference velocity model $v_{0}(\mathbf{r})$ is denoted as $P_{0}\left(\mathbf{r}_{r}, \mathbf{r}_{s}, \omega\right)$, while the first-order Born wavefield is written as $P_{B}\left(\mathbf{r}_{r}, \mathbf{r}_{s}, \omega\right)$.

The phase and amplitude perturbations of the scattered field with respect to the reference field are derived by separation of the real and imaginary parts of the exponential function in equation 1 . Hence, the phase delay resulting from the presence of a velocity anomaly is given by

$$
\Delta \varphi\left(\mathbf{r}_{r}, \mathbf{r}_{s}, \omega\right)=\mathfrak{I}\left(\frac{P_{B}}{P_{0}}\left(\mathbf{r}_{r}, \mathbf{r}_{s}, \omega\right)\right),
$$

which yields for the traveltime residual $\Delta t$

$$
\Delta t\left(\mathbf{r}_{r}, \mathbf{r}_{s}, \omega\right)=\frac{1}{\omega} \Im\left(\frac{P_{B}}{P_{0}}\left(\mathbf{r}_{r}, \mathbf{r}_{s}, \omega\right)\right),
$$

where we have used the linear expression $\Delta \varphi=\omega \Delta t$.

Similarly, the amplitude $A_{R}\left(\mathbf{r}_{r}, \mathbf{r}_{s}, \omega\right)$ of the Rytov wavefield with respect to the amplitude $A_{0}\left(\mathbf{r}_{r}, \mathbf{r}_{s}, \omega\right)$ of the reference wavefield is given by

$$
\ln \left(\frac{A_{R}}{A_{0}}\left(\mathbf{r}_{r}, \mathbf{r}_{s}, \omega\right)\right)=\Re\left(\frac{P_{B}}{P_{0}}\left(\mathbf{r}_{r}, \mathbf{r}_{s}, \omega\right)\right) .
$$

Let the amplitude difference be defined as $\Delta A=A_{R}-A_{0}$. For small perturbations of the amplitude of the Rytov wavefield, the logarithm to leading order is $\ln (1+x) \approx x$ for $x \ll 1$. The amplitude variation with respect to the reference wavefield is approximated then as

$$
\frac{\Delta A}{A_{0}}\left(\mathbf{r}_{r}, \mathbf{r}_{s}, \omega\right) \approx \Re\left(\frac{P_{B}}{P_{0}}\left(\mathbf{r}_{r}, \mathbf{r}_{s}, \omega\right)\right)
$$

for $\Delta A / A_{0} \ll 1$.

Aki and Richards (1980) and Snieder and Lomax (1996) derived the first Born wavefield (from now on, simply called the Born wavefield), which is a solution to the acoustic-wave equation for a constant density and in the presence of a velocity perturbation. The Born wavefield is given by

$$
P_{B}\left(\mathbf{r}_{r}, \mathbf{r}_{s}, \omega\right)=\int_{\mathbf{V}} \frac{2 \Delta v(\mathbf{r}) \omega^{2}}{v_{0}^{3}(\mathbf{r})} P_{0}\left(\mathbf{r}, \mathbf{r}_{s}, \omega\right) G\left(\mathbf{r}_{r}, \mathbf{r}, \omega\right) d \mathbf{V},
$$

where the velocity perturbation with respect to a homogeneous reference velocity $v_{0}(\mathbf{r})=v_{0}$ is denoted as $\Delta v(\mathbf{r})$, and the $2 \mathrm{D}$ integra- 
tion is indicated by $\mathbf{V}$. The 2D far-field Green's function for the homogeneous reference medium is given by (Snieder and Lomax, 1996)

$G\left(\mathbf{r}_{r}, \mathbf{r}, \omega\right)=-\sqrt{\frac{v_{0}}{8 \pi \omega\left|\mathbf{r}_{r}-\mathbf{r}\right|}} \exp i\left(\frac{\omega\left|\mathbf{r}_{r}-\mathbf{r}\right|}{v_{0}}+\frac{\pi}{4}\right)$.

As shown previously (Spetzler and Snieder, 2001), equation 7 can be used to express the Born wavefield in equation 6 explicitly in the system coordinates. For a point source, this yields

$$
\begin{aligned}
P_{B}\left(\mathbf{r}_{r}, \mathbf{r}_{s}, \omega\right)= & \frac{\omega}{4 \pi v_{0}^{2}} \exp i\left(\frac{\omega L}{v_{0}}+\frac{\pi}{4}\right) \\
& \times \int_{-\infty}^{\infty} \int_{0}^{L} \Delta v(\mathbf{r}) \frac{\exp i\left(\frac{\omega L r^{2}}{2 v_{0} z(L-z)}+\frac{\pi}{4}\right)}{\sqrt{z(L-z)}} d z d r .
\end{aligned}
$$

The paraxial approximation that $r \ll z, L-z$ is used to obtain the Born approximation above. A sketch of the system coordinates is shown in Figure 1. Let the point source be located at the origin; the receiver position is placed on the $z$-axis at $z=L$, and the position of the scatterer point is $(z, r)$. The Born wavefield in equation 8 is then divided by the point source solution

$$
P_{0}\left(\mathbf{r}_{r}, \mathbf{r}_{s}, \omega\right)=-\sqrt{\frac{v_{0}}{8 \pi \omega\left|\mathbf{r}_{r}-\mathbf{r}_{s}\right|}} \exp i\left(\frac{\omega\left|\mathbf{r}_{r}-\mathbf{r}_{s}\right|}{v_{0}}+\frac{\pi}{4}\right),
$$

where $\left|\mathbf{r}_{r}-\mathbf{r}_{s}\right|=L$, in order to obtain the monochromatic traveltime delay in equation 3 and the monochromatic amplitude variation in equation 5 .

Recorded waves are never monochromatic, but have broadband frequency properties. The monochromatic traveltime delay and relative amplitude variation are therefore integrated over the frequency band $\left[f_{0}-\Delta f ; f_{0}+\Delta f\right]$. If the broadband frequency characteristics of recorded wavefields with a normalized amplitude spectrum that is, $\int_{f_{0}-\Delta f}^{f_{0}+\Delta f} \mathcal{A}(f) d f=1$ - are taken into account, the broadband traveltime shift for source position $\mathbf{r}_{s}$ and receiver position $\mathbf{r}_{r}$ is given by

$$
\Delta t\left(\mathbf{r}_{r}, \mathbf{r}_{s}\right)=\int_{-\infty}^{\infty} \int_{0}^{L} \Delta v(z, r) K_{\Delta t}^{2 D}(z, r) d z d r
$$

with the frequency-averaged sensitivity function (also known as the Fréchet kernel) for traveltime residuals defined as

$$
K_{\Delta t}^{2 D}(z, r)=-\sqrt{\frac{L}{v_{0}^{5} z(L-z)}} \times \int_{f_{0}-\Delta f}^{f_{0}+\Delta f} \mathcal{A}(f) \sqrt{f} \sin \left(\frac{f \pi L r^{2}}{v_{0} z(L-z)}+\frac{\pi}{4}\right) d f,
$$

which was originally derived by Spetzler and Snieder (2001). Identically, the broadband relative amplitude variation is given by

$$
\frac{\Delta A}{A_{0}}\left(\mathbf{r}_{r}, \mathbf{r}_{s}\right)=\int_{-\infty}^{\infty} \int_{0}^{L} \Delta v(z, r) K_{\Delta A}^{2 D}(z, r) d z d r
$$

with the Fréchet kernel for amplitude variations defined as

$$
\begin{aligned}
K_{\Delta A}^{2 D}(z, r)= & -2 \pi \sqrt{\frac{L}{v_{0}^{5} z(L-z)}} \\
& \times \int_{f_{0^{-}}-\Delta f}^{f_{0^{+}} \Delta f} \mathcal{A}(f) f^{3 / 2} \cos \left(\frac{f \pi L r^{2}}{v_{0} z(L-z)}+\frac{\pi}{4}\right) d f .
\end{aligned}
$$

The frequency-averaged traveltime shifts and amplitude variations for the Rytov wavefield in a 3D medium can be derived similarly. In three dimensions, the monochromatic Green's function for the acoustic wave equation with constant density and velocity is given by

$$
G\left(\mathbf{r}_{r}, \mathbf{r}, \omega\right)=-\frac{\exp i \frac{\omega\left|\mathbf{r}_{r}-\mathbf{r}\right|}{v_{0}}}{4 \pi\left|\mathbf{r}_{r}-\mathbf{r}\right|} .
$$

Equation 14 is used to express the direct wavefield between the source position $\mathbf{r}_{s}$ and the receiver position $\mathbf{r}_{r}$, and the scattered wavefield from the point source to the scatterer point $\mathbf{r}$ to the receiver position (see Figure 1).

For the broadband traveltime shift $\Delta t$, this leads eventually to

$$
\Delta t\left(\mathbf{r}_{r}, \mathbf{r}_{s}\right)=\int_{-\infty}^{\infty} \int_{-\infty}^{\infty} \int_{0}^{L} \Delta v(r, \phi, z) K_{\Delta t}^{3 D}(r, \phi, z) r d z d \phi d r
$$

The Fréchet kernel for traveltime residuals in equation 15 is given by

$$
\begin{aligned}
K_{\Delta t}^{3 D}(r, \phi, z)= & -\frac{L}{v_{0}^{3} z(L-z)} \\
& \times \int_{f_{0}-\Delta f}^{f_{0^{+}}+\Delta f} \mathcal{A}(f) f \sin \left(\frac{f \pi L r^{2}}{v_{0} z(L-z)}\right) d f,
\end{aligned}
$$

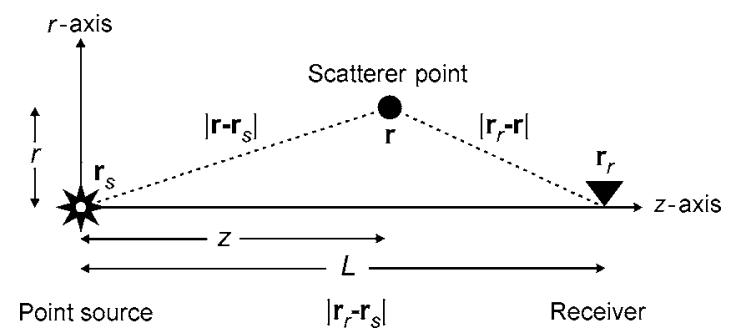

Figure 1. Illustration of the geometric variables to describe the single-scattering process. 
(Hung et al., 2001; Spetzler et al., 2002). In addition, the broadband relative amplitude variation equals

$$
\frac{\Delta A}{A_{0}}\left(\mathbf{r}_{r}, \mathbf{r}_{s}\right)=\int_{-\infty}^{\infty} \int_{-\infty}^{\infty} \int_{0}^{L} \Delta v(r, \phi, z) K_{\Delta A}^{3 D}(r, \phi, z) r d z d \phi d r
$$

with the sensitivity function for relative amplitude variations written as

$$
\begin{aligned}
K_{\Delta A}^{3 D}(r, \phi, z)= & -\frac{2 \pi L}{v_{0}^{3} z(L-z)} \\
& \times \int_{f_{0}-\Delta f}^{f_{0}^{+}+\Delta f} \mathcal{A}(f) f^{2} \cos \left(\frac{f \pi L r^{2}}{v_{0} z(L-z)}\right) d f .
\end{aligned}
$$

An expression similar to equation 18 is derived by Dahlen and Baig (2002) from the Born wavefield.

\section{Properties of the 2D and 3D wave-diffraction theory}

The presented single-scattering wave theory for traveltime delays is a natural extension of the well-known ray theory that provides a high-frequency solution for the elastic wave equation, e.g., Červený (2001). It follows for both $2 \mathrm{D}$ and $3 \mathrm{D}$ scattering theory that

$$
\lim _{f \rightarrow \infty} \Delta t_{\text {scat }}\left(\mathbf{r}_{r}, \mathbf{r}_{s}\right) \approx-\int_{0}^{L} \frac{\Delta v(z)}{v_{0}^{2}} d z=\Delta t_{\text {ray }}\left(\mathbf{r}_{r}, \mathbf{r}_{s}\right),
$$

where the coordinate system in Figure 1 is used. A derivation of a similar expression can be found in Spetzler and Snieder (2001, equa- a)

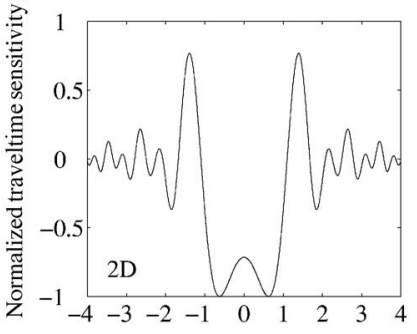

c)

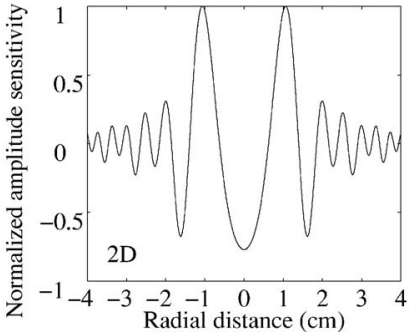

b)

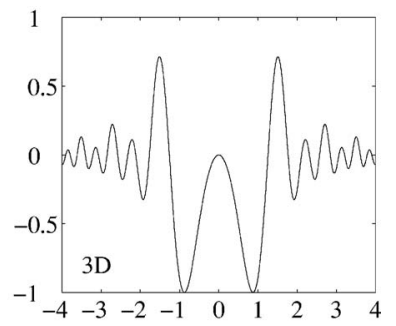

d)

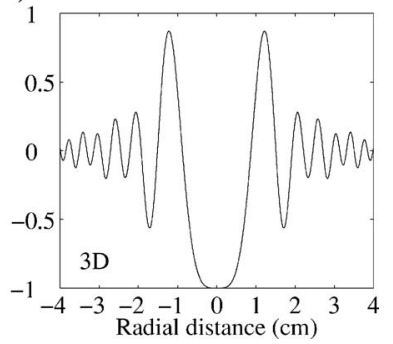

Figure 2. Cross sections of normalized 2D and 3D Fréchet kernels for the traveltime delay and amplitude variations. (a) 2D sensitivity kernel for traveltime shifts; (b) 3D sensitivity kernel for traveltime residuals; (c) 2D Fréchet kernel for amplitude variations; (d) 3D Fréchet kernel for amplitude variations. tion 29). Ray theory predicts that the amplitude of a propagating wavefield is modified by transmission/reflection effects, geometric spreading of the wavefield, and attenuation resulting from energy loss. Following an approach similar to the one taken to arrive at equation 19, one finds that the single-scattering wave theory (equations 12 and 17 for the amplitude attribute) yields the ray theoretical result for infinite-frequency waves. Hence, again using the coordinate system in Figure 1, it follows that the amplitude ratio in the high-frequency limit is given by

$$
\lim _{f \rightarrow \infty}\left[\frac{\Delta A}{A_{0}}\left(\mathbf{r}_{r}, \mathbf{r}_{s}\right)\right]_{\mathrm{scat}} \approx \frac{1}{2 L v_{0}} \int_{0}^{L} z(L-z) \frac{\partial^{2} \Delta v(z)}{\partial r^{2}} d z,
$$

where $\partial^{2} \Delta v(z) / \partial r^{2}$ is the second derivative of the velocity perturbation field perpendicular to the reference raypath - in this case, a straight line joining the source and receiver points because of the constant reference velocity. Expression 20 resembles equation 30 of Neele et al. (1993) who worked with ray-perturbation theory, as well as equation 170 of Dahlen and Baig (2002) who used a higher-order expansion of the velocity-perturbation field to deduce the high-frequency behavior of the amplitude part of finite-frequency waves. Notice that equation 20 is derived using ray-perturbation theory similar to Neele et al. (1993). This implies that formally, the infinite frequency amplitude formulation in equation 20 , is valid only in smooth velocity-perturbation media.

Ray theory (Červený, 2001) is valid in media with anomalies of a size $a$ larger than the wavelength and the width $L_{F}$ of the first Fresnel volume (from now on, simply called the Fresnel volume); hence,

$$
\lambda / a<1 \text { and } L_{F} / a<1 .
$$

Physically speaking, the Fresnel volume is the region between the source and receiver for which the scattered wavefield contributes constructively at the receiver position. According to Spetzler and Snieder (2001), at a distance $z$ from a point source, the width $L_{F}(z)$ of the 2D Fresnel volume for a source-receiver distance $L$ (see Figure 1) is given by

$$
L_{F}^{2 D}(z)=\sqrt{\frac{3 \lambda z(L-z)}{L}},
$$

whereas for 3D media,

$$
L_{F}^{3 D}(z)=\sqrt{\frac{4 \lambda z(L-z)}{L}} .
$$

Notice that $L_{F}^{3 D}(z=L / 2)>L_{F}^{2 D}(z=L / 2)$. As a result of the paraxial approximation, expressions 22 and 23 are approximately accurate away from the source and receiver. However, the single-scattering wave theory does not introduce any limitation on the size of the anomaly relative to the Fresnel volume.

The cross sections perpendicular to the direction of propagation of the normalized Fréchet kernels for traveltime delays in equations 11 and 13 and for relative amplitude variations in equations 16 and 18 are illustrated in Figure 2. The source-receiver distance $L=20 \mathrm{~cm}$; the constant reference velocity $v_{0}=1480 \mathrm{~m} / \mathrm{s}$. The frequency integration is performed analytically over the frequency band $f-\Delta f=300 \mathrm{kHz}$ and $f+\Delta f=600 \mathrm{kHz}$, for which it is assumed that $\mathcal{A}(f)=1 /(2 \Delta f)$ (Spetzler et al., 2002). The cross sections of the sensitivity kernels are computed at the midpoint 
between the source and receiver (i.e., $z=L / 2$ ) where the sensitivity kernels have the maximum width. The figures show normalized sensitivity to velocity perturbations with respect to traveltime and amplitude variations as a function of the perpendicular distance from the source-receiver line which, in the ray theoretical sense, is equal to the geometric ray path between the source and receiver. Wave diffraction theory predicts that the frequency-averaged traveltime shift is maximum for scatterers located off the geometric ray. The sensitivity to slowness perturbations decays away from the geometric ray path because of the integration over a broad frequency band. The area of most significant sensitivity is equivalent to the Fresnel volume (Spetzler and Snieder, 2001).

For wave propagation in a 3D medium, the traveltime delay is insensitive to velocity perturbations on the source-receiver line (Figure $2 b$ ). This differs from ray theory that predicts a nonzero sensitivity only for velocity anomalies intersecting with the geometric ray path. For 2D wave propagation, the ray theoretical prediction is also different from the finite-frequency wave result because the sensitivity is maximally away from the geometric ray (Figure 2a). Both the 2D and 3D Fréchet kernels for amplitude variations have a maximum sensitivity to a velocity anomaly on the geometric ray, whereas the sidelobes outside the Fresnel volume decay away from the ray, again, because of broadband frequency integration (Figure $2 \mathrm{c}$ and d).

\section{THE 3D ULTRASONIC WAVE EXPERIMENT}

The experimental setup used for the velocity perturbation measurements is shown schematically in Figure 3. Both source and receiver are suspended in a stiff frame. A positioning system is used to enable translation of the receiver perpendicular to the $z$-axis. The stationary source is a piezoelectric, spherically focused $500-\mathrm{kHz}$ transducer (Panametrics, type V389) with a focal distance of approximately $6 \mathrm{~cm}$ in water. The focal point acts as a point source. The receiver (indicated by triangles in Figure 3 ) is a broadband needle hydrophone with a tip diameter of $1 \mathrm{~mm}$ (SEA, type PZT-Z441000). Source, receiver, and sample are placed in a 1-cubic-meter tank filled with tap water.

To act as a perturbation of the homogeneous velocity field, small spheres with various diameters $d$ made from synthetic rubber $(d=10 \mathrm{~mm})$ and teflon $(d=7.5 \mathrm{~mm})$ are used. For the spheres to remain stationary during the measurements, they are suspended in a Plexiglas frame using 0.25 -mm-thick fishing line. Teflon has a negative velocity contrast $(1275 \mathrm{~m} / \mathrm{s})$, and rubber has a positive velocity contrast $(1514 \mathrm{~m} / \mathrm{s})$, compared to water at room temperature $( \pm 1485 \mathrm{~m} / \mathrm{s})$. The propagation velocities of teflon and rubber were determined during separate traveltime measurements on cylindrical samples from which the spheres were manufactured at a later stage.

For the velocity sensitivity measurements, the following procedure is applied. First, the sphere is placed on the axis between the source (i.e., the focal point) and central receiver position. The center of the sphere is $10 \mathrm{~cm}$ from both the source and central receiver. Next, the field is scanned along the receiver line as indicated in Figure 3. The receiver separation is $1 \mathrm{~mm}$, whereas the receiver array extends to $\pm 5 \mathrm{~cm}$ on both sides of the central receiver. The procedure is repeated in absence of the sphere so that the traveltime differences and the relative amplitude variation between the two data sets can be determined for each individual receiver location.
The recorded (perturbed and reference) data sets are bandpass filtered between 232 and $720 \mathrm{kHz}$, as the $\mathrm{S} / \mathrm{N}$ ratio is the highest for this range. The traveltime residuals and the relative amplitude variation are determined from the perturbed and reference traces using a spectral-ratio approach. Let the reference waveform in the frequency domain be given by

$$
d_{\text {ref }}(\omega)=A_{\text {ref }}(\omega) \exp \left(i \omega t_{\text {ref }}\right) S_{\text {ref }}(\omega),
$$

and the perturbed waveform is written as

$$
d_{\text {pert }}(\omega)=A_{\text {pert }}(\omega) \exp \left(i \omega t_{\text {pert }}\right) S_{\text {pert }}(\omega) .
$$

Note that the source wavelets $S_{\text {ref }}(\omega)=S_{\text {pert }}(\omega)$ in the ultrasonic experiment. The amplitude factor $A(\omega)$ includes transmission effects, the geometric spreading factor, and attenuation inherent to energy losses. The ratio of the perturbed and reference wavefield splits into an amplitude part and a traveltime delay part:

$$
\frac{d_{\text {pert }}}{d_{\text {ref }}}(\omega)=\frac{A_{\text {pert }}}{A_{\text {ref }}}(\omega) \exp (i \omega \Delta t),
$$

which is a complex number. The angle of the wavefield ratio in the complex plane divided by the angular frequency gives the observed traveltime shift:

$$
\Delta t(\omega)=\frac{1}{\omega} \angle\left(\frac{d_{\text {pert }}}{d_{\text {ref }}}(\omega)\right) .
$$

The relative amplitude variation $\Delta A / A_{\text {ref }}(\omega)=A_{\text {pert }} / A_{\text {ref }}(\omega)-1$ [where $A_{\text {pert }}(\omega)=A_{\text {ref }}(\omega)+\Delta A(\omega)$ ] is retrieved from the norm of the ratio between the perturbed and reference waveform. Hence,

$$
\frac{\Delta A}{A_{\text {ref }}}(\omega)=\left\|\frac{d_{\text {pert }}}{d_{\text {ref }}}(\omega)\right\|-1 .
$$

The broadband frequency characteristics of the two waveforms are included by averaging the monochromatic traveltime delay in equation 27 and the relative amplitude variation in equation 28 over the associated frequency interval.

Equations 15 and 17 are used to compare the experimental results with $3 \mathrm{D}$ theory. Again, the condition $\int_{f_{0}-\Delta f}^{f_{0}+\Delta f} \mathcal{A}(f) d f=1$ is used to normalize the experimentally determined spectra in equations 16 and 18 where $\mathcal{A}(f)$ corresponds to the spectrum of the trace recorded at the receiver position under investigation. In the ray theoretical case, the traveltime delay is computed from linear ray theory in equation 19,

\section{Crossline experiment}
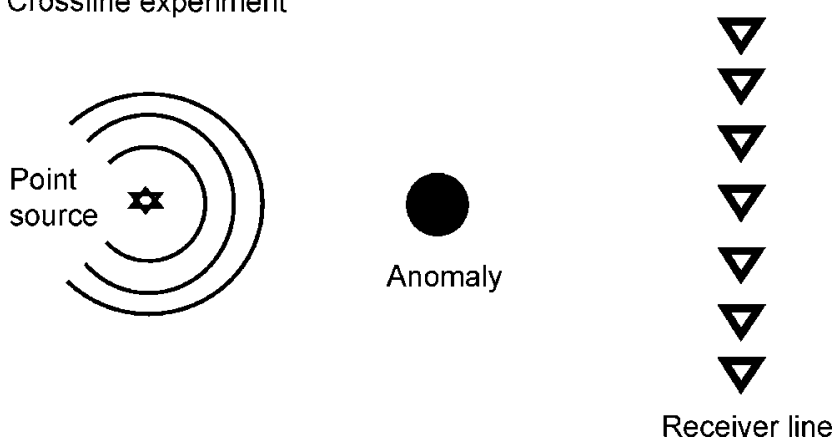

Figure 3. Schematic (top) view of the setup used for the sensitivity tests. For each test, two complementary scans are performed: one with and one without an anomaly present between source and receiver. 
as well as from nonlinear ray theory, including ray-bending effects in the perturbed velocity field. The ray theoretical relative-amplitude variation inherent to the geometric spreading factor is computed using equation 20 .

Figure 4 shows the recorded wavefields in the presence of the teflon and rubber spheres. For the teflon sphere (Figure 4a), a clear triplication (i.e., area of high acoustic energy) occurs in the observed waveform data. The presence of the triplication indicates that strong ray-bending effects are encountered. In Figures 5-7, the traveltime delay and amplitude variation for the wavefields in Figure 4 are shown. We plotted the equivalent results based on linear and nonlinear ray theory, together with the exact results for a point-source wavefield propagating in a homogeneous background medium and incident upon an elastic sphere.

The exact results were calculated using the theory presented by Hickling (1962). Using a spherical coordinate system centered in the anomaly, the wavefield $P_{i}(\omega)$ emanating from a point source at a distance $r_{0}$ can be expressed as

$$
P_{i}(\omega)=i k P_{0} \sum_{n=0}^{\infty}(2 n+1)(-1)^{n} P_{n}(\cos \theta) j_{n}(k r) h_{n}\left(k r_{0}\right),
$$

where $P_{n}$ is the $n$th order Legendre polynomial; $j_{n}$ and $h_{n}$ are spherical Bessel and Neumann functions, respectively; $k$ is the fluid wavenumber; and $r$ is the distance between the origin of the sphere and the receiver location. The scattered field resulting from the point-source

a)

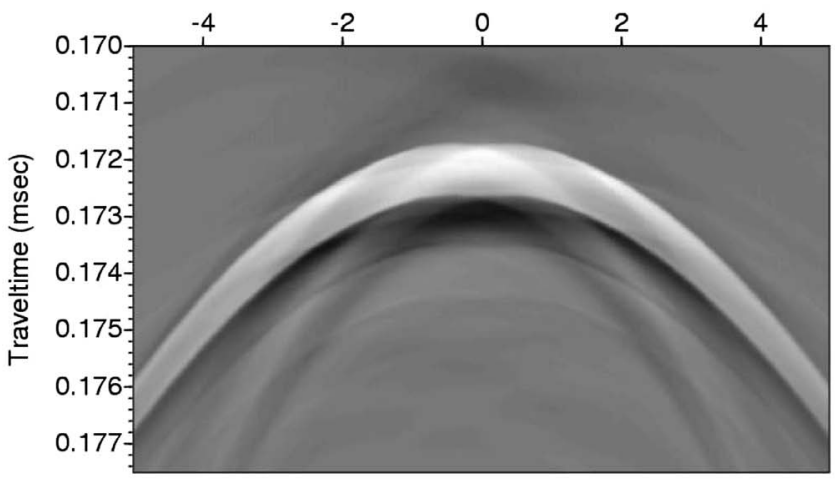

b)

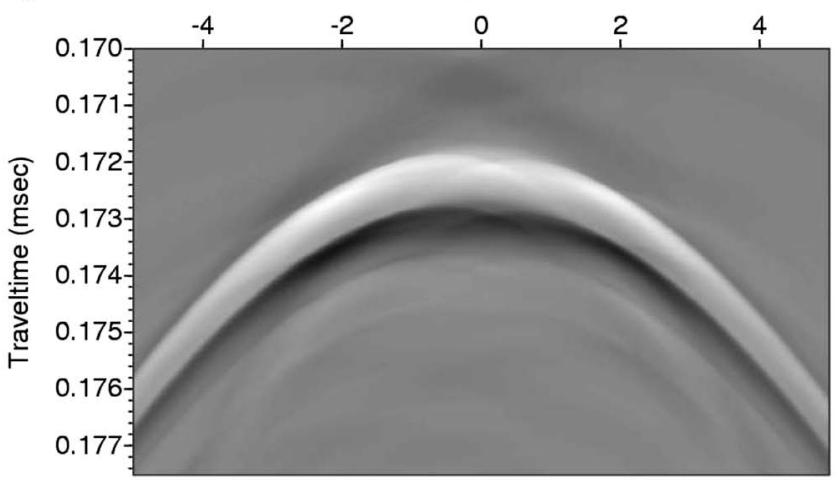

Figure 4. The recorded wavefields in the presence of (a) negative(teflon) velocity perturbation and (b) positive-(rubber) velocity perturbation. Notice the triplication resulting from the negative velocity difference at the midpoint of the horizontal axis in Figure 4a.
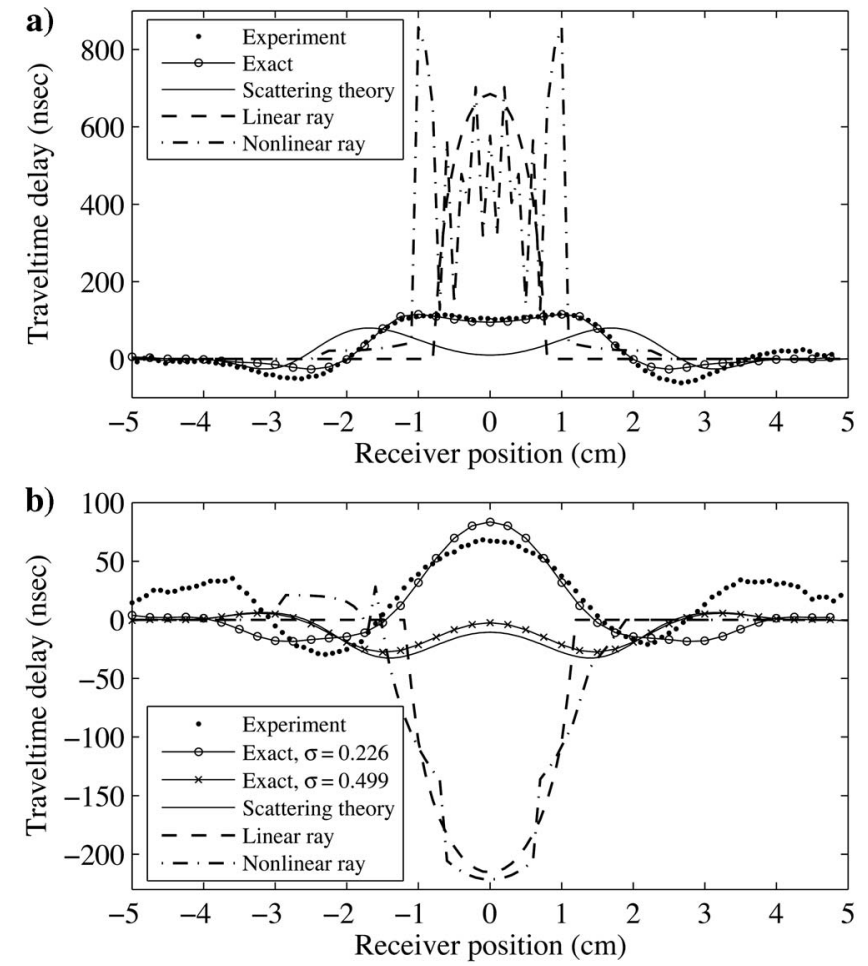

Figure 5. Comparison between the experimentally observed traveltime delays and the predicted ones using finite-frequency wave theory and ray theory. (a) $\lambda / a=0.4, L_{F}^{3 D} / a=3.2$, and $\Delta v / v=-13.5 \%$ (i.e., teflon). (b) $\lambda / a=0.3, L_{F}^{3 D} / a=2.4$, and $\Delta v / v=2.7 \%$ (i.e., rubber).
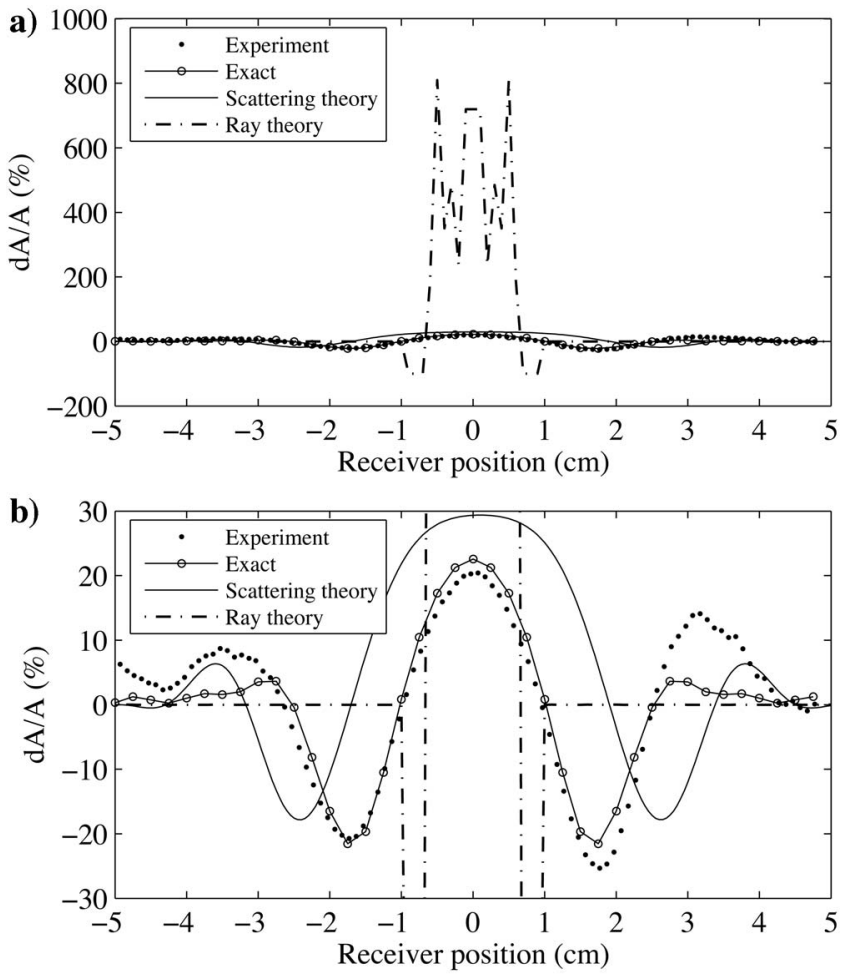

Figure 6. (a) Similar to Figure 5 except for the relative amplitude variation in the case of teflon. (b) A close-up view of (a). 
wavefield incident upon an elastic sphere is expressed as

$$
P_{s}(\omega)=P_{0} \sum_{n=0}^{\infty} c_{n} h_{n}(k r) P_{n}(\cos \theta),
$$

where coefficients $c_{n}$ follow from the appropriate boundary conditions at the surface of the sphere, i.e., (1) continuity of fluid pressure and normal component of the stress in the solid at the interface; (2) continuity of normal displacement of solid and fluid at the interface; and (3) vanishing of shear stress in the solid at the interface. Among other parameters, coefficients $c_{n}$ contain information on the elastic sphere, such as density $\rho$ and Poisson ratio $\sigma$ (through which the shear wave velocity is determined). The $\sigma$ and $\rho$, both absent in the linear-scattering theory, are determined independently from the ultrasonic measurements. For teflon, we found $\rho=2157 \mathrm{~kg} / \mathrm{m}^{3}$ and $\sigma=0.413$ (i.e., $c_{s}=491 \mathrm{~m} / \mathrm{s}$ ), whereas for rubber, $\rho=1020 \mathrm{~kg} / \mathrm{m}^{3}$ and $\sigma=0.23$ (i.e., $c_{s}=896 \mathrm{~m} / \mathrm{s}$ ). The total field external to the sphere $P_{t o t}(\omega)$ is given by the sum of the incident field and the scattered field:

$$
P_{t o t}(\omega)=P_{i}(\omega)+P_{s}(\omega) .
$$

Applying and averaging equation 31 over the frequency interval $232-720 \mathrm{kHz}$, the results can be compared immediately with the curves in Figures 5-7 to study the influence of the limitations of linear scattering theory in relation to the absence of shear-wave and density contributions.

For the teflon anomaly, the traveltime delay and relative amplitude variations resulting from the velocity contrast between the sphere and water are shown in Figures 5a and 6a and 6b. In general, the time delay and fractional amplitude distortion predicted by ray theory are several times larger in magnitude than the experimentally observed values. Also, the width of the ray theoretical curves is smaller than what is observed in the ultrasonic experiment. The 3D finite-frequency wave theory yields better results than ray theory when predicting the observed traveltime shift and amplitude ratio, both in magnitude and shape of the experimental curves. However, there is still a clear mismatch between the $3 \mathrm{D}$ finite-frequency wave theory and the experimental observations as a result of the triplication present in the recorded waveform data. The 3D finite-frequency wave theory is linear and, in turn, does not include strong nonlinear ray-bending and multipathing effects that are associated with the formation of triplications.

For the rubber measurements (see Figures $5 b$ and 7 ), the agreement between linear theory and experiment has improved, compared to the teflon results. Again, ray theory predicts values too large for the traveltime shifts and relative amplitude variation. On the other hand, the linear scattering theory underestimates the traveltime delay and, to a lesser extent, the amplitude variation as observed during the experiments. This is because of the small difference in compressional wave velocity between fluid and rubber, in addition to the omission of shear-wave effects in linear scattering theory. Comparing the scattering theoretical result for rubber with the exact result in the absence of a shear wave (i.e., $c_{s} \approx 0 \mathrm{~m} / \mathrm{s}$ or $\sigma \approx 0.5$ ), it is observed that both curves are very similar. However, when a shear wave is introduced into the exact solution by decreasing the value for the Poisson ratio to the experimentally determined value of $\sigma=0.23$, the exact result increasingly agrees with the experimental results, including the characteristic uprise in the relative amplitude variation around the central receiver position (Figure 7b). In conclusion, in the case of rubber, the $\mathrm{P}$-wave velocity perturbation between fluid and elastic solid is so small that the shear wave in the sphere is the dominating event. Because shear waves are unaccounted for in the presented linear theory, traveltime and amplitude effects are underestimated compared to the experimental observations (though still predicted considerably better than by linear and nonlinear ray theory). In practice, this will rarely pose a problem, for a small contrast in compressional wave velocities between two elastic media usually will go together with a small contrast in shear-wave velocity, contrary to the case discussed above for an elastic sphere in an acoustic (i.e., shearless) background medium.

Finally, regarding Figures 6 and 7, a simple computation of the total transmission coefficient

$$
T_{t o t}=4 \frac{\rho_{w} v_{w} \rho_{s p h} v_{s p h}}{\left(\rho_{w} v_{w}+\rho_{s p h} v_{s p h}\right)^{2}},
$$

at the interfaces between the spheres (the density and P-wave velocity are denoted as $\rho_{s p h}$ and $\left.v_{s p h}\right)$ and the surrounding fluid ( $\rho_{w}$ and $v_{w}$ are the density and sound speed of water) of the incident and outgoing wavefield for the center receiver position reveals that the amplitude loss is on the order of $15 \%$ for teflon and $0.5 \%$ for rubber because of the impedance differences between background and anomaly. However, Figures 6 and 7 show that the ray theoretical results are several times larger than the observed values. This is caused by the presence of the second-derivative operator in equation 20 that acts to make effects of small-scale structures on ray-theoretical amplitudes very prominent. In reality, the wavefield is subject to amplitude variations on the order of $15 \%$ for both the teflon and rubber anomaly because of the effect of finite-frequency waves.
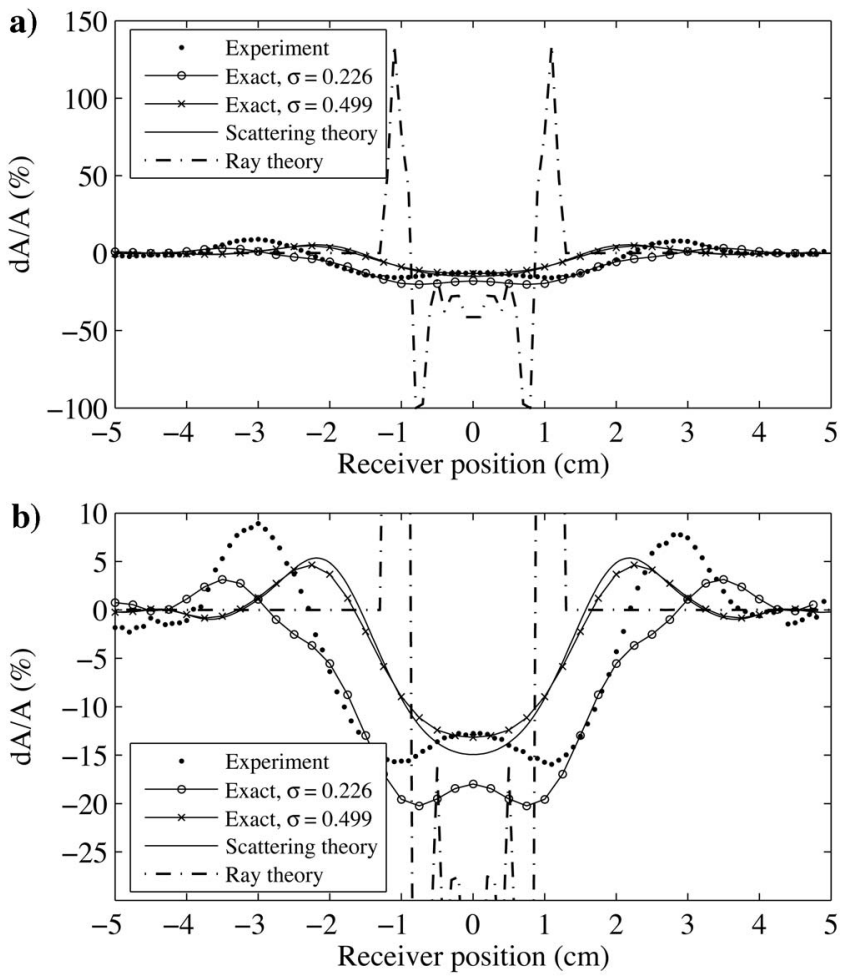

Figure 7. (a) Similar to Figure 5 except for the relative amplitude variation for the rubber anomaly. (b) A close-up view of (a). 


\section{D NUMERICAL SIMULATIONS OF THE ULTRASONIC WAVE EXPERIMENT}

The finite-frequency wave theory tests in the ultrasonic laboratory experiment are restricted to a few case studies of different velocity perturbations with respect to the velocity of sound in water. Beyond teflon and rubber, we are aware of no other appropriate materials. That is why a numerical finite-difference (FD) solution of the elastic-wave equation is used to mimic the ultrasonic laboratory experiment for a large range of different velocity contrasts. The FD simulations are similar to the ultrasonic wave experiment with the exception of the dimensionality (2D instead of 3D). The source wavelet has a broadband characteristic with the central frequency fixed at $500 \mathrm{kHz}$. The source is modeled as a Ricker wavelet. The geometry for this numerical test is identical to the sketch in Figure 3, and the 3D ultrasonic experimental parameters are repeated. Hence, a 2D, spherical (cylindrical) wavefield is emitted from a point source, and the total wavefield is recorded along an array of receivers perpendicular to the line going through the source position and the center of the anomaly. Sets of perturbed and reference data are obtained by respectively including and omitting the spherical anomaly in the velocity model. Numerical results are obtained for anomaly velocities $v_{\text {pert }}$ equal to $1260 \mathrm{~m} / \mathrm{s}, 1360 \mathrm{~m} / \mathrm{s}, 1600 \mathrm{~m} / \mathrm{s}$, and $1700 \mathrm{~m} / \mathrm{s}$, whereas the reference velocity $v_{\text {ref }}$ is fixed at $1480 \mathrm{~m} / \mathrm{s}$. The velocity contrasts between the anomaly and the reference velocity are then $-15 \%,-8 \%, 8 \%$, and $15 \%$, in which the case of the $-15 \%$ velocity anomaly is comparable to the teflon experiment. For all computations, the diameter of the spherical anomaly is $7.5 \mathrm{~mm}$.

Similar to the experimental data processing, the traveltime shifts and the amplitude variation inherent to the velocity anomaly are obtained using the previously discussed spectral-ratio approach. The theoretical traveltime delays and amplitude fluctuations are computed with 2D finite-frequency wave theory in accordance with equations 10 and 12, as well as with linear and nonlinear ray theory,
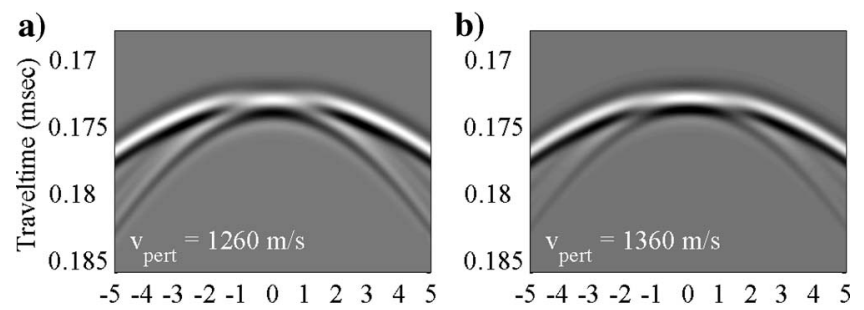

c)
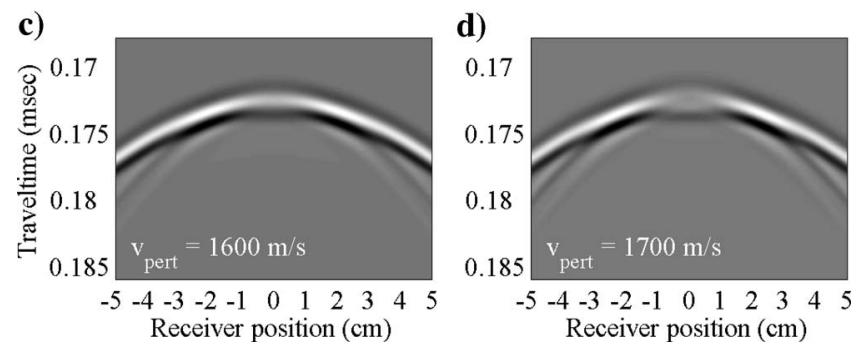

Figure 8. Numerical FD results for a range of velocity perturbations. (a) $\Delta v / v=-15 \%$; (b) $\Delta v / v=-8 \%$; (c) $\Delta v / v=8 \%$; and (d) $\Delta v / v=15 \%$. In (a), a single caustic is generated at the central receiver position because of a strong negative-velocity perturbation (i.e., $-15 \%)$. In (d), two triplications resulting from a large positivevelocity difference (i.e., 15\%) are seen clearly on both sides of the central receiver position. which takes into account ray bending resulting from velocity contrasts.

Gaussian beam theory (Červený, 2001) offers an alternative way to account for the finite-frequency effects of waves. We have tested the Gaussian beam theory as described in Hill $(1990 ; 2001)$ in the 2D numerical experiment. The time delays computed with Gaussian beam theory do not differ significantly from the time shifts obtained with the nonlinear two-point ray tracer. For the relative amplitude variation computation, Gaussian beam theory returns maximum values of $\pm 0.5 \%-1 \%$ of amplitude variation for the $\pm 15 \%$ velocity-contrast experiments.

In Figure 8, synthetic FD results are shown for a range of velocity contrasts between $-15 \%$ and $15 \%$ with respect to the propagation velocity in water (i.e., $v_{\text {ref }}=1480 \mathrm{~m} / \mathrm{s}$ ). The triplication observed previously in Figure 4a is clearly visible also in Figure 8a. The other case of caustic formation is illustrated in Figure 8d, where two caustic points away from the center receiver line are observed. Figure $8 b$ and $\mathrm{c}$ shows less significant signs of triplications because of the weaker velocity perturbations.

Similar to the 3D case, the 2D diffraction theory leads to significantly better results compared to linear and nonlinear ray theory (see Figures 9-11). In general, the traveltime shifts and amplitude ratios calculated using ray theory are too large in magnitude, and the widths of the curves are too narrow, compared to the numerically observed values. The high-frequency oscillations in the nonlinear traveltime delays are caused again by strong ray-bending and multipathing effects. The $2 \mathrm{D}$ wave-diffraction theory predicts relative amplitude changes in the order of magnitude of $\pm 40 \%$. To a lesser extent, this is observed also in the experimental 3D data (Figures 6 and 7). Notice the small discrepancy between the traveltime residual and relative amplitude variation predicted by wave-scattering theory and the numerically observed ones in Figures 9b, 9c, and $11 b$ and 11c. In these figures, nonlinear ray-bending effects resulting from the formation of triplications are still weak, justifying the use of finite-frequency wave theory. In Figures 9a, 9d, 11a, and 11d, there might be a)

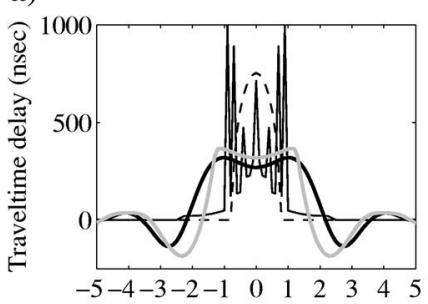

c)

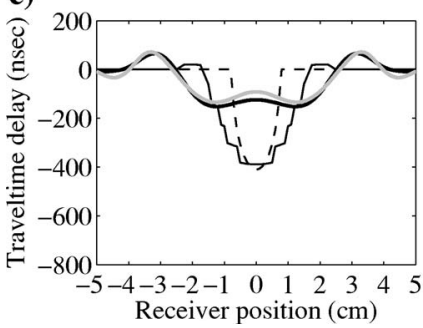

b)

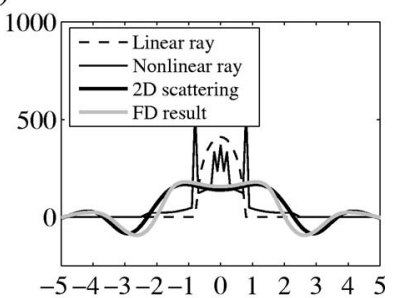

d)

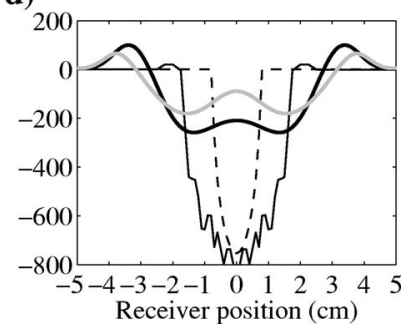

Figure 9. Comparison between the numerically observed traveltime delays and the predicted ones compiled with finite-frequency wave theory and ray theory. The experimental parameters are $\lambda / a=0.4$ and $L_{F}^{2 D} / a=2.8$. The range of velocity contrasts are (a) $\Delta v /$ $v=-15 \%$; (b) $\Delta v / v=-8 \%$; (c) $\Delta v / v=8 \%$; and (d) $\Delta v / v=15 \%$. 
severely bent rays going through caustics that are not included in the theoretical formulation. Consequently, we are at the limit of the validity of the single-scattering wave approach.

\section{SEISMIC APPLICATIONS}

Although not perfect, finite-frequency kernels have the potential to improve resolution of seismic imaging experiments at low computational costs. While in seismology, these benefits are still overshadowed by incomplete data coverage (Van der Hilst and De Hoop, 2005; Trampert and Spetzler, 2006), the seismic applications clearly suffer less from lack of data. This is illustrated by two examples of finite-frequency wave theory in seismic imaging experiments. The two applications that we discuss are (1) traveltime tomography using transmission data and (2) amplitude recovery of reflected data prior to AVO/AVA inversion.

In the first example, waveform data from the Durham laboratory experiment are used (Legget et al., 1993; Pratt, 1999). In this experiment, ultrasonic transmitted waves are recorded in a crosswell configuration with two vertical wells. The velocity structure is manufactured with epoxy. Figure 12a illustrates schematically the velocity model that consists of small and large structures, compared to the maximum Fresnel width (i.e., $L_{F}$ ). However, the separate layers in the physical model are not that homogeneous, as indicated in the figure, nor is the contact surface between the layers completely free of tiny air bubbles because the mixing of the resins had to be done quickly (Neil Goulty, personal communication, 2003). According to equation 21 , the requirements for ray theory are not satisfied for the low-velocity half-cylinder. On the contrary, the conditions for the application of the finite-frequency wave theory are valid for the whole velocity model in the Durham experiment. In addition, the ultrasonic transmission experiment includes realistic noise contributions because of uncertainties in source-receiver positions, in the traveltime estimation, and in the estimation of the reference velocity (Legget et al., 1993).

In the ultrasonic experiment, $500-\mathrm{kHz}$ piezoelectric transducers are used to emit and record waveform data. Fifty-one sources and receivers give a total of 2601 measured broadband waveforms. The physical dimensions for the crosswell velocity structure are $46.5 \mathrm{~mm}$ in the offset direction and $125 \mathrm{~mm}$ in the depth direction.
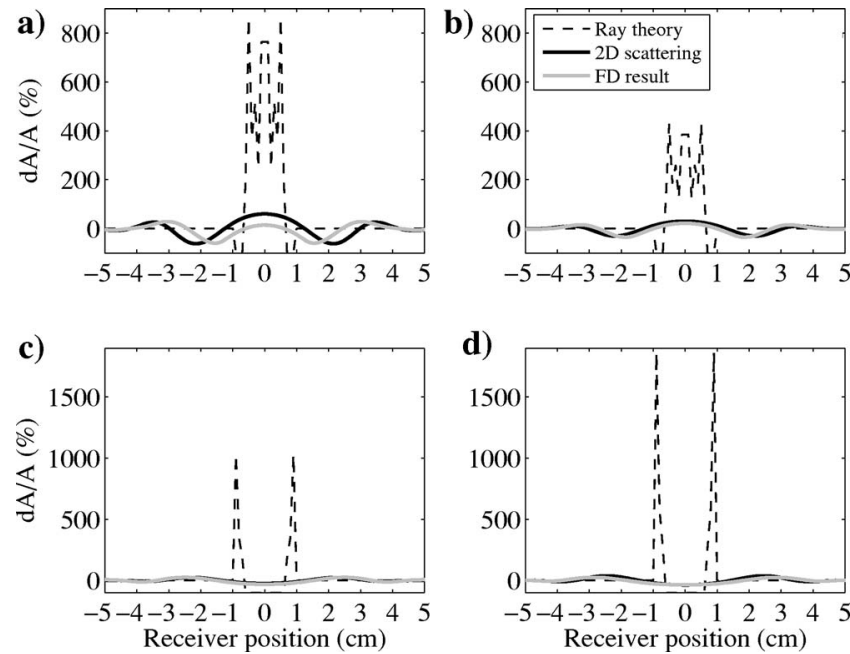

Figure 10. Similar to Figure 9 except for relative amplitude variations.
To simulate a realistic crosswell measurement with frequencies between 200 and $500 \mathrm{~Hz}$, all distances, times, and frequencies are scaled by a factor 1000. In turn, the lateral length and depth of the area between the two wells have the dimensions $46.5 \times 125 \mathrm{~m}$. The target zone for the inversion is the half-cylinder-shaped, low-velocity anomaly and the vertical fault below. The reference velocity model is a layered velocity structure without the fault between the lowest two layers. A detailed description of the tomographic inversion of the acoustic data using both finite-frequency scattering theory and ray theory can be found in Spetzler (2003).

The inverted velocity model, compiled with the finite-frequency wave theory and ray theory, is presented in Figure $12 \mathrm{~b}$ and c, respectively. The solid lines indicate the interfaces between the homogeneous layers of resin. The low-velocity half-cylinder between 60 and $70 \mathrm{~m}$ depth is resolved best in the velocity model inverted with the first-order finite-frequency wave theory. The strength of the low-velocity contrast of the half-cylinder is underestimated as well as smoothed out in Figure 12c. This is a consequence of the limitations of the high-frequency approximation in ray theory. In both inversion
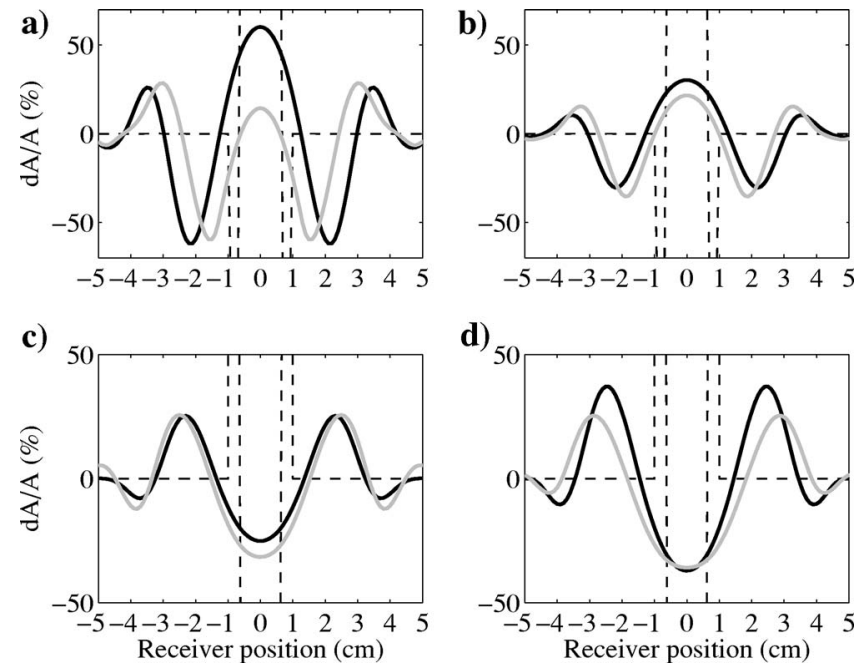

Figure 11. A close-up view of Figure 10.

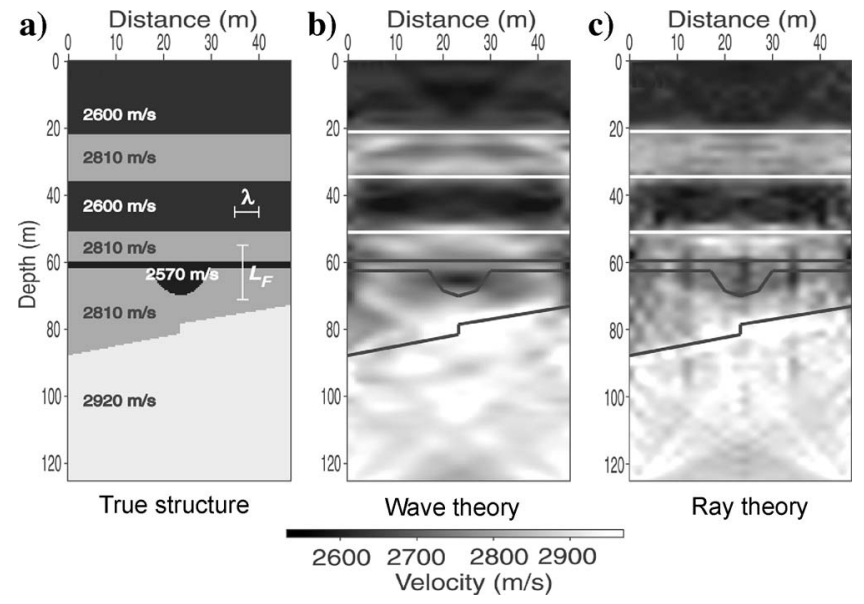

Figure 12. Crosswell tomography. (a) Schematic velocity structure. (b) Velocity cross section compiled with the finite-frequency wave theory. (c) Velocity cross section generated with ray theory. 
results, there seems to be an imprint of the small, vertical fault at approximately $80 \mathrm{~m}$ depth.

The finite-frequency wave tomographic inversion does not require extra hardware or memory than is necessary for a similar inversion approach based on ray theory. The two inversions in this example were carried out on a laptop with an Intel Pentium IV, 3.2$\mathrm{GHz}$ processor with $512 \mathrm{mb}$ memory running on Suse linux 9.1. For the construction of the forward-modeling matrix for a grid parametrization of $24 \times 64=1536$ grid cells and 2601 data points, it takes $20 \mathrm{CPU}$ minutes for the finite-frequency wave approach and $1 \mathrm{CPU}$ minute for ray theory. The tomography inversion for both approaches takes 5 CPU minutes.

The second example is a numerical-reflection seismic experiment. A gas-saturated overburden anomaly (also known as a gas chimney) is located over an interface dipping $5^{\circ}$. The $\mathrm{P}$-wave velocity model is illustrated in Figure 13a. The seismic parameters in the top layer are $v_{p}=2000 \mathrm{~m} / \mathrm{s}, v_{s}=1200 \mathrm{~m} / \mathrm{s}$, and $\rho=1100 \mathrm{~kg} / \mathrm{m}^{3}$, whereas in the bottom layer, $v_{p}=3000 \mathrm{~m} / \mathrm{s}, v_{s}=1300 \mathrm{~m} / \mathrm{s}$, and $\rho=1500 \mathrm{~kg} / \mathrm{m}^{3}$. The gas-saturated overburden perturbation is modeled with a Gaussian function. The maximum velocity contrast is $130 \mathrm{~m} / \mathrm{s}$ with respect to the homogeneous reference velocity in the top layer, and the standard deviation of the overburden anomaly is $100 \mathrm{~m}$ in both the horizontal and vertical directions. As illustrated on the Schlumberger seismic glossary web page (i.e., www.glossary. oilfield.slb.com), a gas chimney may have a significant attenuating effect on P-P stacks. An additional example of transmission distortions as large as $70 \%$ on prestack seismic reflected waves inherent to a)

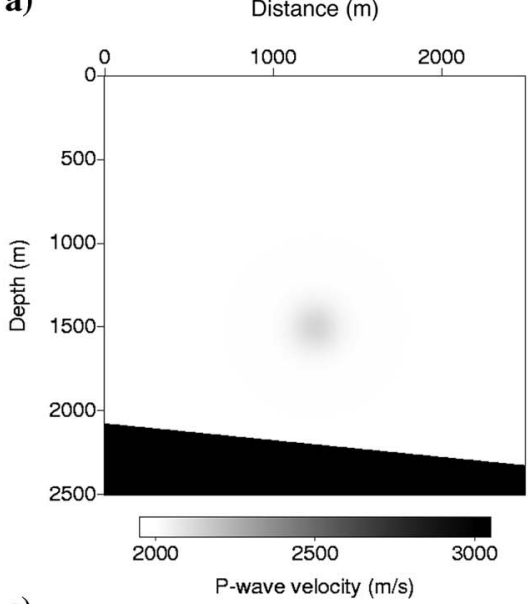

c)

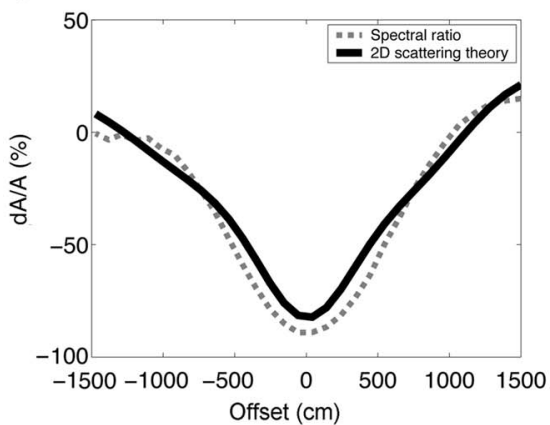

b)

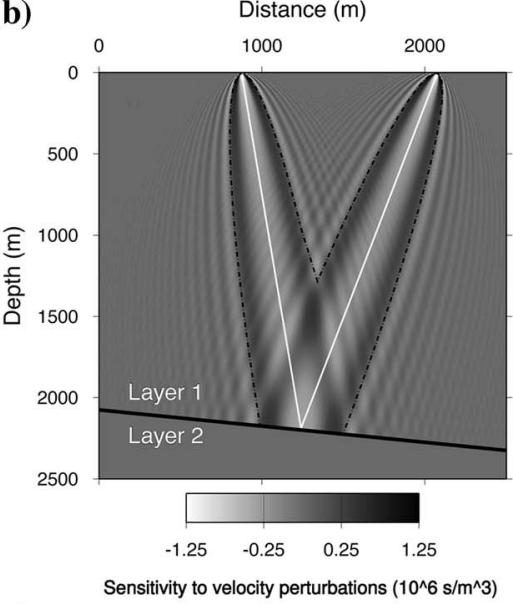

d)

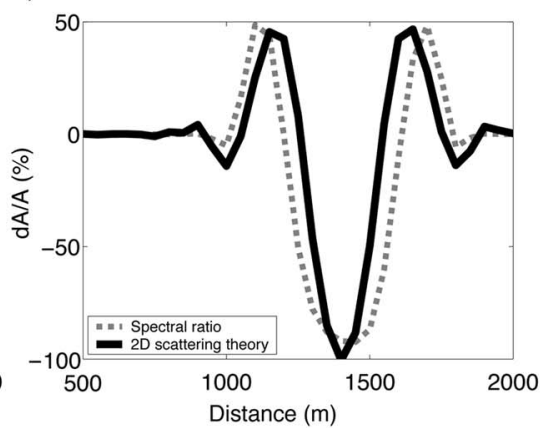

Figure 13. Propagation effect resulting from a gas-saturated overburden anomaly on the amplitude of reflection seismic data. (a) Velocity structure; (b) Fréchet kernel for a reflected wavefield; (c) CMP gather, and (d) near-offset gather. fault structures in the Gulf of Mexico is found in Hatchell (2000). This numerical example shows that the attenuation of the amplitude of a P-P reflection below a gas-saturated overburden anomaly can be explained by single-scattering wave theory.

The finite-frequency sensitivity kernel for a reflected wave with the frequency content between $20-60 \mathrm{~Hz}$ is shown in Figure $13 \mathrm{~b}$. Two-way wavefield theory is used to decompose the Fréchet kernel into down- and upgoing parts which are computed with first-order diffraction theory. The gray-scale color illustrates the sensitivity to velocity perturbations. The straight white line indicates the raypath, and the white dashed line marks the boundary for the Fresnel volume for the central frequency of $40 \mathrm{~Hz}$. The width of the Fresnel volume above the interface is on the order of $500 \mathrm{~m}$, which is several times larger in size than the overburden anomaly.

A full, elastic, finite-difference modeling experiment was applied to compute reference and perturbed reflection data, respectively, without and including the gas-saturated overburden anomaly. The spectrum of the reflection data is dominant between 20 and $60 \mathrm{~Hz}$. A common midpoint (CMP) gather for the CMP at $1250 \mathrm{~m}$ (below the gas chimney) and a near-offset gather were constructed from the simulated reflection data. The relative amplitude variation resulting from propagation through the overburden perturbation was obtained from the spectral ratio of the perturbed and reference gathers. The theoretical relative amplitude was computed with $2 \mathrm{D}$ wave diffraction theory for reflected waves. In Figure $13 \mathrm{c}$ and d, the observed and computed values are compared. For both gathers, the amplitude is attenuated by $80 \%-90 \%$, solely as a result of the presence of the gassaturated anomaly. Hence, the finite-frequency wave theory offers a way to correct for amplitude losses resulting from overburden anomalies in CMP gathers and near-offset gathers. Afterward, the amplitude-corrected gathers can be used for further estimation of structure and seismic parameters.

\section{CONCLUSIONS}

Finite-frequency wave propagation in media with structures smaller in size than the Fresnel volume leads to the counterintuitive result that the sensitivity to traveltime shifts is maximally away from the geometric ray and that the sensitivity for the amplitude is maximum on the geometric ray for both $2 \mathrm{D}$ and $3 \mathrm{D}$. Remarkably, for wave propagation in $3 \mathrm{D}$, the sensitivity for traveltime shifts is zero on the ray path because of wavescattering effects. Those findings are in contradiction with standard ray theory. By means of ultrasonic wavefield experiments, these results are validated successfully for a relevant range of anomaly sizes and velocities for which the regime of ray theory is invalid. The findings in the ultrasonic experiment are supported by numerical simulations. The counterintuitive results from finite-frequency, wave-scattering theory and the associated laboratory experiments can be explained physically by the principle of wavefield interference. Monochromatic waves diffracted by velocity contrasts interfere with other monochromatic components in the field. Adding up 
constructively at the receiver, these waves compose the recorded broadband wavefield.

First-order, finite-frequency wave theory works well for anomaly diameters which are approximately one-third of the Fresnel volume width and for velocity differences up to $\pm 15 \%$ in the reference velocity. This is concluded from 2D numerical simulations in which there are no constraints on the magnitude of the velocity contrast. For stronger velocity contrasts, nonlinear effects related to the formation of caustics dominate the problem.

To improve the resolution of seismic imaging experiments, adequate wave-propagation theory should be applied. The finite-frequency wave theory has immediate use in several such experiments, and its regime goes beyond the standard ray theory. We have discussed two possible applications to illustrate how to incorporate the finite-frequency wave theory in seismic imaging experiments. Another possible application of the first-order diffraction theory is to compute the phase and amplitude operators in the wavefield-extrapolation part of migration experiments.

\section{ACKNOWLEDGMENTS}

This manuscript is sponsored by the European Community through the Atlass project NNE5-1999-20211 and by the Dutch Technology Foundation STW through project DAR.6293. The manuscript has been improved because of the thorough work of an anonymous reviewer. We appreciate the time and effort that this reviewer invested in our study of finite-frequency wave theory. We gratefully acknowledge Neil Goulty and Gerhard Pratt for permission to use the Durham University laboratory data.

\section{REFERENCES}

Aki, K., and P. G. Richards, 1980, Quantitative seismology, theory and methods: Freeman and Company.

Beydoun, W. B., and A. Tarantola, 1988, First Born and Rytov approximations: Modeling and inversion conditions in a canonical example: Journal of the Optical Society of America, 83, 1045-1055.

Born, M., and E. Wolf, 1959, Principles of optics: Pergamon Press.

Červený, V., 2001, Seismic ray theory: Cambridge University Press.

Dahlen, F., and A. Baig, 2002, Fréchet kernels for body-wave amplitudes:

Geophysical Journal International, 150, 440-466.
De Hoop, A. T., 1985, Time-domain far-field scattering of planer scalar waves in the Born approximation: Journal of the Optical Society of America, 2, 1961-1964.

De Wolf, D. A., 1967, Validity of Rytov's approximation: Journal of the Optical Society of America, 57, 1057-1058.

Hatchell, P. J., 2000, Fault whispers: Transmission distortions on prestack seismic reflection data: Geophysics, 65, 377-389.

Hickling, R., 1962, Analysis of echoes from a solid elastic sphere in water: Journal of the Acoustical Society of America, 34, 1582-1592.

Hill, N. R., 1990, Gaussian beam migration: Geophysics, 55, 1416-1428. , 2001, Prestack Gaussian-beam depth migration: Geophysics, 66, 1240-1250.

Hung, S., F. Dahlen, and G. Nolet, 2001, Wavefront healing: A bananadoughnut perspective: Geophysical Journal International, 146, 289-312.

Legget, M., N. R. Goulty, and J. E. Kragh, 1993, Study of traveltime and amplitude time-lapse tomography using physical model data: Geophysical Prospecting, 415, 599-619.

Marquering, H., F. A. Dahlen, and G. Nolet, 1999, Three-dimensional sensitivity kernels for finite-frequency traveltimes: The banana-doughnut paradox: Geophysical Journal International, 137, 805-815.

Neele, F., J. C. VanDecar, and R. Snieder, 1993, A formalism for including amplitude data in tomographic inversions: Geophysical Journal International, 115, 482-496.

Pratt, G. R., 1999, Seismic waveform inversion in the frequency domain, Part 1: Theory and verification in a physical scale model: Geophysics, 64, 888901

Rytov, S. M., Y. A. Kravtsov, and V. I. Tatarskii, 1989, Principles of statistical radiophysics 4: Wave propagation through random media: Springer Verlag.

Snieder, R., and A. Lomax, 1996, Wavefield smoothing and the effect of rough velocity perturbations on arrival times and amplitudes: Geophysical Journal International, 125, 796-812.

Spetzler, J., 2003, Comparison of ray theory and finite-frequency wave theory in crosswell tomography: 65th Annual Meeting EAGE, Extended Abstracts, F40.

Spetzler, J., and R. Snieder, 2001, The effect of small-scale heterogeneity on the arrival time of waves: Geophysical Journal International, 145, 786-796.

, 2004, Tutorial: The Fresnel volume and transmitted waves: Geophysics, 169, 653-663.

Spetzler, J., C. Sivaji, O. Nishizawa, and Y. Fukushima, 2002, A test of ray theory and scattering theory based on a laboratory experiment using ultrasonic waves and numerical simulations by finite-difference method: Geophysical Journal International, 148, 165-178.

Trampert, J., and J. Spetzler, 2006, Surface wave tomography: Finite frequency effects lost in the null space: Geophysical Journal International, 164, 394-400.

Van der Hilst, R. D., and M. V. De Hoop, 2005, Banana-doughnut kernels and mantle tomography: Geophysical Journal International, 163, 956-961.

Woodward, M. J., 1992, Wave-equation tomography: Geophysics, 57, 15-26.

Zhao, L., T. Jordan, and C. Chapman, 2000, Three-dimensional Fréchet differential kernels for seismic delay times: Geophysical Journal International, 141, 558-576. 